Association française pour l'étude des corps gras - 39es Journées Chevreul, Paris - 21 et 22 novembre 2001

Oléagineux, Corps Gras, Lipides. Volume 8, Numéro 2, 133, Mars - Avril 2001, La filière

ARTICLE

Génomique et filière oléagineuse

Programme

Mercredi 21 novembre 2001

Introduction

Jean-Luc PERRIN, Unilever, Président de I'AFECG

Génomique et lipides

Présentation générale de la génomique

Michel CABOCHE, Président de Génoplante

Métabolisme des lipides de la plante

Michel DELSENY

Génoplante : rôle, structure et devenir

Michel BOUCLY, Président de l'AMSOL

Conférence Chevreul: Bilan des travaux sur le colza

Michel RENARD, Directeur de recherches Inra

Génomique et nutrition humaine

Biodisponibilité et ANC

Nicole COMBE, ITERG

Connaissance dans le domaine de la génomique humaine appliquée à la nutrition

Francis QUETIER, Génoscope

Table ronde avec les trois intervenants et Stéphane PASTEAU, Monsanto

Jeudi 22 novembre 2001

Génomique et nutrition animale

Besoins en alimentation animale

Anne BOURDILLON, Glon-Sanders

Génomique animale et métabolisme [en cours]

La réponse de la génomique 
Michèle CHAMPION, Limagrain

Génomique et production non alimentaire

Les besoins en lipochimie

Alfred WESTFECHTEL, Cognis (Allemagne)

Acides gras d'intérêt industriel obtenus par génie génétique

J.C. KADER, Jussieu

Réglementation, expertise, mise sur le marché

\section{Conférence Chevreul}

Réglementation/expertise/mise sur le marché

M. MACLEOD (RU)

Les enjeux de la protection intellectuelle : brevets, COV

Roland PETIT-PIGEARD, Sicasov

Réglementation Nouveaux Aliments

Hervé REVERBORI, DGAL

\section{Conclusions}

André POUZET, Cetiom, vice-Président de l'AFECG

\section{Contact :}

Véronique Fabrien-Soulé - Fax : 33146371560

$<$ v.fabien-soule@fncg.fr> 